Journal of Geophysics and Engineering 2010, vol.7, pp.395-403

doi:10.1088/1742-2132/7/4/006

\title{
Wavelet-preserved PP- and PS-wave registration
}

\author{
Feng Zhang and Yanghua Wang \\ Centre for Reservoir Geophysics, Department of Earth Science and Engineering, Imperial College \\ London, SW7 2BP, UK
}

\begin{abstract}
Calibration of PP- and PS-wave reflection events is a crucial step in multicomponent seismic data inversion and quantitative analysis. This paper presents a workflow of calibration including an optimized estimation of the P- to S-wave velocity ratio and wavelet preservation after a PS-wave trace transformation to the PP-wave two-way time. The optimized velocity ratio is obtained from the spectral analysis of correlation coefficients versus perturbations of a time-variant velocity-ratio function. This analysis may also be conducted in a target-oriented fashion which involves not only searching for the additive perturbation but also the gradient of the local velocity ratio, to overcome possible ambiguities in the correlation spectrum. However, when a PS-wave trace is transformed from the PS time to the PP time, the PS reflection wavelets along the trace are compressed. This wavelet distortion needs be removed to preserve the original PS-wave frequency content, before further inversion processing. Wavelet compression means stretching in the frequency spectrum, and such spectral stretching is time variant depending upon the local P- to S-wave velocity ratio. Thus, the restoration is implemented on the time-frequency spectrum in the Gabor transform domain. Thereafter, wavelet-preserved PS-wave reflections, presented in PP time, may be used in a PP- and PS-wave joint inversion.
\end{abstract}

Keywords: multicomponent seismic, event calibration, wavelet preservation, time-frequency spectrum, Gabor transform

\section{Introduction}

When using multi-component seismic data for imaging, inversion and interpretation, the calibration between the PP-wave reflections and the converted PS-wave events is a crucial step in the workflow. It transforms PS-wave reflection events from PS-wave time to the corresponding PP-wave time, and is often implemented as an iterative process in which transformed PS-wave events are correlated with the PP-wave reflections to verify the selection of the P- to S-wave velocity ratio. In this paper, we present an event calibration procedure which not only transforms a PS-wave trace to the PP-wave two-way time, but also preserves the original wavelets in the transformed PS-wave trace.

The PP- and PS-wave calibration is also called event registration in multicomponent data analysis. Behle and Dohr (1985) implemented a combined velocity analysis between stacked PP-waves and converted PS-waves for correlation. Garotta (1985) developed a 
cross-correlation method for P-wave and S-wave sections within small windows using a guessed time scale factor. Gaiser (1996) analyzed two cross-correlation methods with general application to determine the ratio of the long-wavelength P-wave velocity to the S-wave velocity. Li et al. (1999) and Zhang and Li (2004) suggested using a PS-wave moveout correction to create a velocity-ratio spectrum similar to the conventional velocity spectral analysis. Ogiesoba and Stewart (2003) developed a velocity analysis procedure using Thomsen's (1999) non-hyperbolic traveltime equation to compute semblance for scanning the depth-varying, zero-offset velocity ratio. Nickel and Sonneland (2004) presented an automatic event registration method under the assumption that for the PP- and PS-wave volumes, only the locations of seismic events exhibit changes, whereas the amplitudes remain the same. While the constant-amplitude assumption is used for registration and is not an assumption of the total workflow, the scheme is indeed effective in practice (Nickel and Sonneland, 2005). Fomel (2007) suggested an event registration method based on local similarity measurement between a PP-wave image and a squeezed or stretched PS-wave image.

In this paper, we develop a perturbation method for the correlation analysis between the PP- and PS-wave events, to estimate the time-variant velocity ratio:

$$
\gamma(t)=\frac{V_{P}(t)}{V_{S}(t)}
$$

where $V_{P}$ and $V_{S}$ are the $\mathrm{P}$ - and $\mathrm{S}$-wave average velocities, respectively, and $t$ is the PP-wave vertical two-way traveltime. We set up an initial $\gamma(t)$ model by selecting the $\gamma$ values from visual comparison between the PP- and PS-wave traces, and update the model based on a correlation analysis with various perturbations. Considering the complexity of field data and sometimes the effect of low signal-to-noise ratio, we also implement the $\gamma$ analysis in a target-oriented fashion for more confident estimation.

During the trace transformation from the PS time to the PP time, the PS-wave reflections are compressed along the time axis. However, time compression also squeezes the wavelets which were convolved with reflection coefficients. Therefore, we suggest a wavelet correction step following the event registration. Ideally, PS- to PP-time transformation should be treated as a kinematic process, and the dynamic information of the original wavelets should be preserved.

As a P-S wavelet is squeezed, the corresponding frequency spectrum is stretched. The correction process attempts to preserve the original frequency content of PS wavelets. We perform a Gabor transform over the compressed PS-wave trace to generate a time-frequency spectrum, and modify the frequency spectrum (both amplitude and phase) for each reference time, based on the local wavelet-compression rate. Then we obtain a wavelet-preserved trace in the time domain by an inverse Gabor transform.

In the following sections, we first justify the wavelet preservation after PS- to PP-time compression. We then present the event calibration and the wavelet preservation in sequence.

\section{Justification for wavelet restoration}

For prestack time migration or normal moveout (NMO) correction where non-zero offset data are time corrected to match with zero offset, there is an NMO stretching effect which is most pronounced at far offsets, due to the travel time following a hyperbolic equation. A similar time-variant compression exists in the PS-wave registration problem discussed in this paper. Basically, event registration is a time to time conversion which maps PS reflections from time $\tau$ to the time $t$. After registration, PP and PS reflections have the same 'pseudo-depth time' 
$t$ for a given reflector. Theoretically, since the PS reflection has a wavelength less than that of the PP reflection, it sees a finer structure than the PP-wave reflection (Gaiser, 1996), if the difference in the S-wave path attenuation could be removed.

Although the higher resolution of PS-wave is beneficial to stratigraphic interpretation, true wavelets in both PP and PS-wave traces without frequency-content distortion are needed in prestack seismic inversion. This is because the frequency dependency of any inverted property is the key for the subsequent fluid discrimination. The original wavelets should be restored from the compressed PS-wave data before seismic inversion. For an introduction of joint prestack inversion of PP- and PS-wave reflection data, we adopt the ray-impedance concept, proposed by Wang (2003). We transform prestack PP- and PS-wave gathers into the ray-parameter domain. For a constant ray parameter, a pair of PP- and PS-wave traces shares exactly the same ray path between the source and the reflection point, and have different ray-paths between the reflection and receiver points. That means the PP- and PS-wave reflection events are from exactly the same reflection point. After the event calibration, these PP and PS-waves from the same reflection point thus have the same depth-type time vertically. Therefore, reflections in a pair of PP and calibrated PS-wave traces with a constant ray parameter correspond to each other sample by sample.

However, the PP-and PS-waves recorded at the surface have different frequency contents. This is due to the effects such as frequency-dependent attenuation, different ray length along different return paths, although PP- and PS-waves share the same ray path from the source to the reflection point. As we do not know the mechanism to correct compressed PS-wavelet to a wavelength that is right for the 'pseudo-depth' time, instead, we attempt to restore the original PS-wavelets before time compression.

After the event registration and wavelet restoration, PP- and PS-waves at the same time $t$ are reflections from the same reflection point. But separate wavelets are needed for the inversion of the PP- and PS-wave data.

\section{Perturbation method for event calibration}

For clarity of presentation, throughout this paper we denote the PP wave vertical two-way time as $t$ and the PS wave two-way time as $\tau$. In the event calibration, a key parameter is the effective $\gamma(t)$ function defined in equation 1 . This velocity ratio function may also be calculated using PP- and PS-wave vertical times $t$ and $\tau$ by

$$
\gamma(t)=\frac{2 \tau-t}{t}
$$

Compressing a PS-wave trace from time $\tau$ to the PP-wave reference time $t$ can be generally represented as

$$
u_{P S}(t)=\Gamma(t, \tau) u_{P S}(\tau)
$$

where $u_{P S}(\tau)$ is the original PS-wave trace in time $\tau, u_{P S}(t)$ is the PS-wave trace in time $t$, and $\Gamma$ is the transform operator. To construct a transformed sample $u_{P S}(t)$, the time $\tau$ is found from equation 2 based on $t$ and $\gamma(t)$. The waveform amplitude around time $\tau$ is built using a sinc function interpolation as

$$
u_{P S}(t)=\sum_{k} u_{P S}(k \Delta \tau) \operatorname{sinc}\left(\frac{\tau(t)}{\Delta \tau}-k\right),
$$


where $\tau(t)=(1+\gamma) t / 2$, and $\Delta \tau$ is the sampling interval of the original PS-wave trace. Therefore, in discrete form where the total number of samples in $u_{P S}(t)$ is $m$ and the total number of samples in $u_{P S}(\tau)$ is $n$, each row of the $m \times n$ matrix $\Gamma$ is a set of coefficients of the sinc function. In examples shown in this paper, the interpolation window is 9 points, and $\Delta \tau=\Delta t, \Delta t$ being the sampling interval of the PP-wave trace.

To evaluate whether the $\gamma(t)$ function is appropriately selected, we estimate the zero-lag correlation coefficient between the compressed PS-wave trace and the reference PP-wave trace as

$$
c(t)=\frac{\sum_{\xi=-T / 2}^{T / 2} u_{P S}(t+\xi) u_{P P}(t+\xi)}{\left[\sum_{\xi=-T / 2}^{T / 2} u_{P S}^{2}(t+\xi) \sum_{\xi=-T / 2}^{T / 2} u_{P P}^{2}(t+\xi)\right]^{1 / 2}},
$$

where $T$ is the correlation window length.

We demonstrate this calibration method using a synthetic seismic data set, for which the model consists of six reflectors. We perform prestack time migration to produce common-image-point (CIP) gathers, presented in the ray-parameter domain, for the purpose of the ray-impedance inversion in the prestack domain (Wang, 2003). We choose a pair of CIP gathers (Figures 1a and 1c) as the example of PP- and PS-wave calibration. We can visually pick the $\gamma$ values in time $\tau$ (Figure 1b), and then squeeze the $\gamma$ curve and the PS-wave CIP gather to the time $t$ to verify whether the $\gamma(t)$ function is appropriately selected (Figures 1d and 1e).

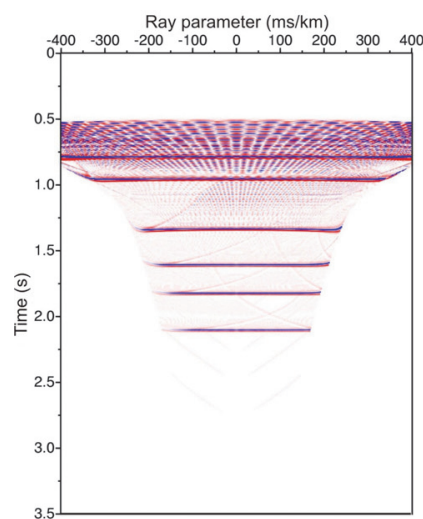

(a)

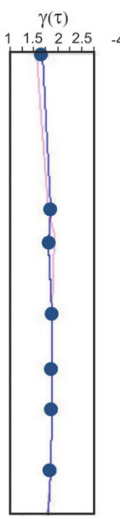

(b)

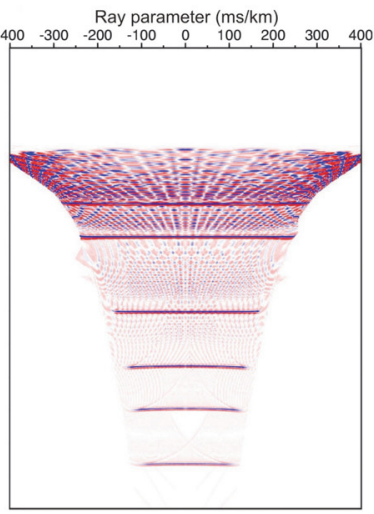

(c)

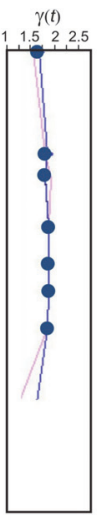

(d)

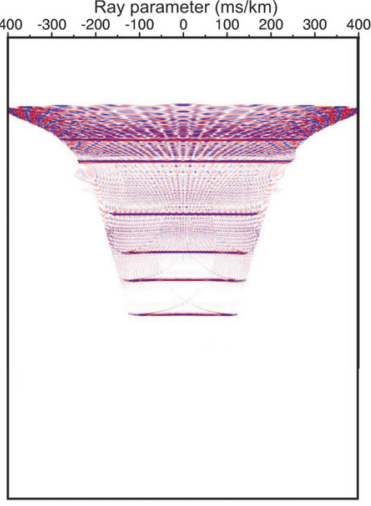

(e)

Figure 1. (a) A PP-wave common-image-point (CIP) gather. (b) The $\gamma(\tau)$ analysis panel, where $\tau$ is the PS-wave vertical two-way time. (c) A PS-wave CIP gather. (d) The $\gamma(t)$ analysis panel, where $t$ is the PP-wave vertical two-way time. (e) Transformed PS-wave CIP gather. The background red curves in (b) and (d) are references from a neighboring CIP gather.

Among the scanning methods based on correlation coefficients, a stable method for estimating time-invariant $\gamma$ is a constant velocity-ratio scanning method (Gaiser, 1996). Given a pair of stacked PP- and PS-wave traces (Figure 2a), the PS-wave trace is transformed with respect to a range of time-invariant $\gamma$ values (Figure $2 b$ ). These transformed traces are correlated sequentially to the reference PP-wave trace, generating a correlation coefficient spectrum (Figure 2c). However, it is difficult to identify the $\gamma$ trend unambiguously from 
this correlation spectrum. For instance, there may be more than one high correlation region at a certain time, and it is difficult to select which is the correct one. An analogous problem occurs with the presence of multiples in conventional velocity spectra displays.

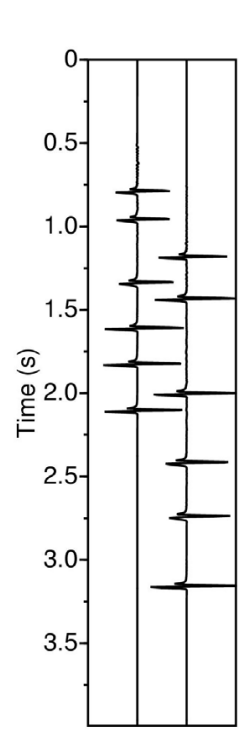

(a)

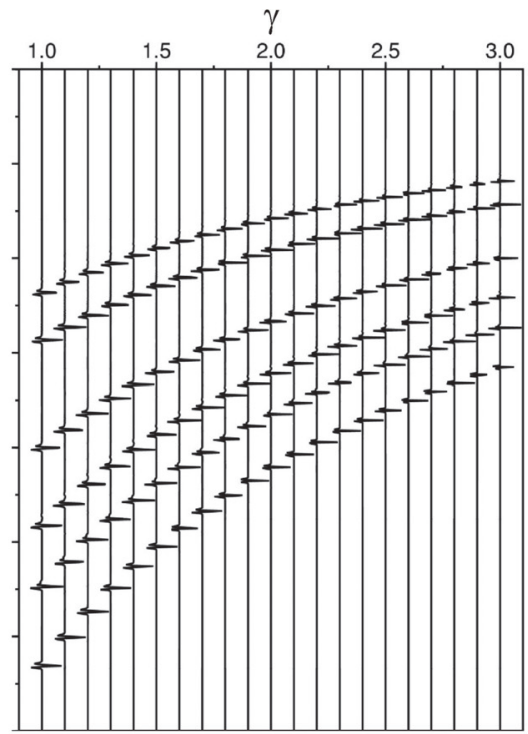

(b)

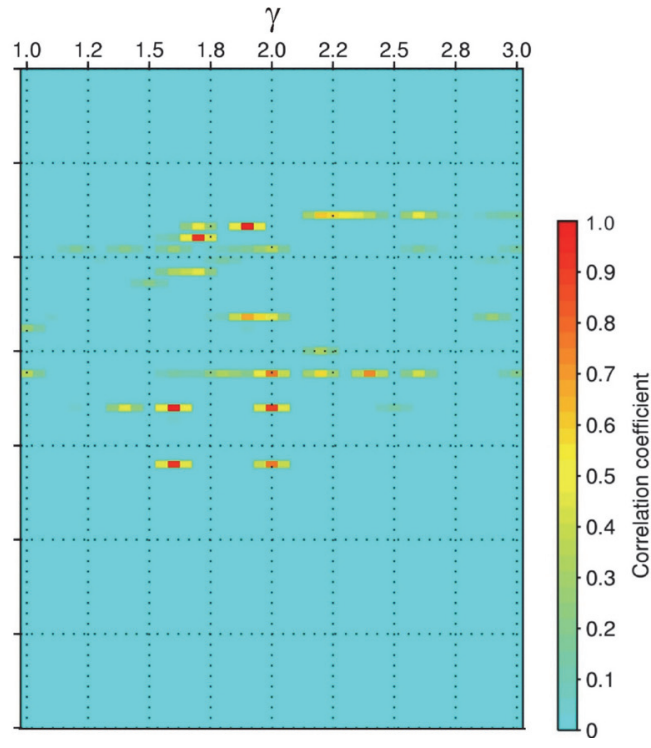

(c)

Figure 2. Scanning for time-invariant $\gamma$. (a) A pair of stacked PP- and PS-wave traces. (b) Transformed PS-wave traces using different constant $\gamma$ values. (c) Zero-leg correlation coefficients between the group of transformed PS-wave traces and the PP-wave trace. It is difficult to pick $\gamma$ values from this correlation spectrum.

Instead, a time-variable $\gamma(t)$ function can be used in scanning. Gaiser (1996) used the P-wave velocity and a linear mudrock relationship (Castagna et al., 1985) for the S-wave velocity to predict the time-variable velocity ratio. Although this method depends upon the assumption of the empirical relationship between the P- and S-wave velocities, using a mudrock relationship can rule out unrealistic cases and thus be effective in finding an initial guess. A practical solution for estimating time-variant $\gamma(t)$ function is selecting $\gamma$ values manually by direct comparison between the PP- and PS-wave gathers, as shown in Figure 1, if there are individual distinct reflectors on the PP- and PS-wave images which can be linked.

Using this time-dependent $\gamma(t)$ as an initial model, we then perform perturbation to find an optimized $\gamma(t)$ function. We generate a group of transformed PS-wave traces (Figure 3a) using time-variable $\gamma(t)$ with additive perturbations between -0.4 and 0.4 (with an interval of 0.04), and calculate the corresponding correlation-coefficient spectrum (Figure 3b), which reveals a very clear $\gamma(t)$ trend for this synthetic example, in which the true value is simply $\gamma(t)=2$ for most time $t$. Based on the correlation analysis, we update the $\gamma(t)$ function (Figure 3c), on which updated points are linked by B-spline interpolation, producing a smooth $\gamma(t)$ function so that the differentiation with respect to time, $\gamma^{\prime}(t)=d \gamma(t) / d t$ exists. Differentiation is needed in the following wavelet preservation.

\section{Wavelet preservation}

After the PS-to-PP time conversion, wavelets are compressed along the transformed PS-wave trace $u_{P S}(t)$. Consequently the band-limited frequency spectrum has been stretched (Figure 
4). This spectrum stretching effect should be removed, so as to recover the wavelets on the original PS-wave trace $u_{P S}(\tau)$.

We implement the wavelet transformation in the Gabor transform domain by means of following three steps: (1) Gabor transforming $u_{P S}(t)$ to generate a time-frequency spectrum; (2) modifying the amplitude spectrum $u_{P S}(\omega, t)$ over all different times $t$; and (3) performing an inverse Gabor transform to produce a time-domain trace $u_{P S}(t)$ with corrected wavelets. As we make the correction based on a modelled $\gamma(t)$ function and not the local spectrum, implementing frequency spectrum correction in the Gabor transform domain may avoid inappropriate distortion related to the geology. A very similar model-based spectrum correction scheme is the Gabor transform domain inverse $Q$ filtering algorithm (Wang, 2006, 2008), which depends on a given attenuation model. This approach may also be applicable to the removal of stretch effects from NMO correction which is an identical problem as we know the times before and after moveout correction and therefore also know the stretch factor.

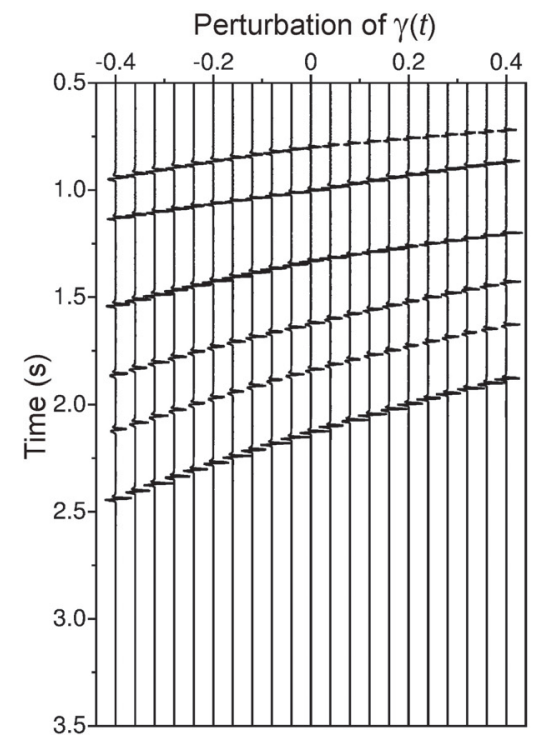

(a)

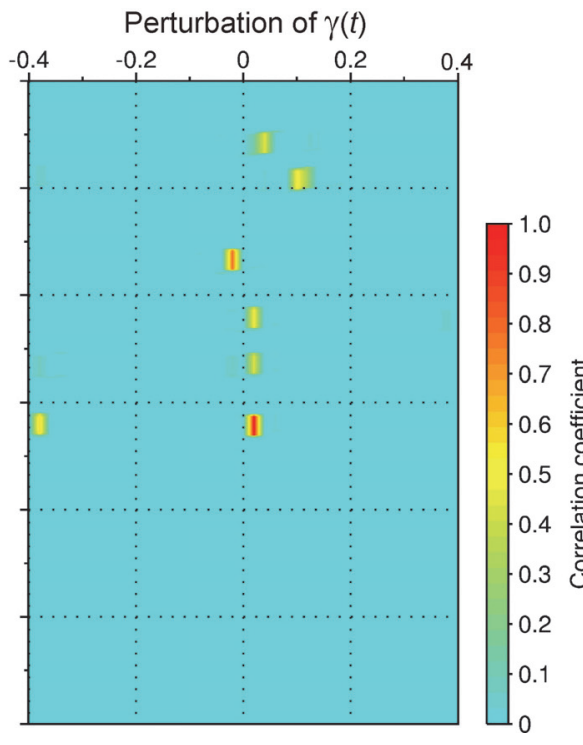

(b)

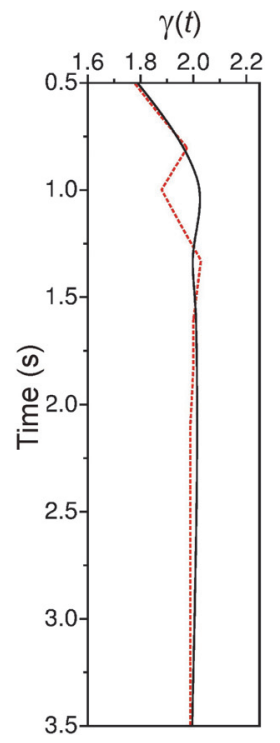

(c)

Figure 3. (a) Transformed PS-wave traces using time-variable $\gamma(t)$ with perturbations. (b) The PPand PS-wave correlation coefficient spectrum. (c) Comparison of the initial $\gamma(t)$ function (dashed red curve) and updated $\gamma(t)$ function (solid black line).

Time domain

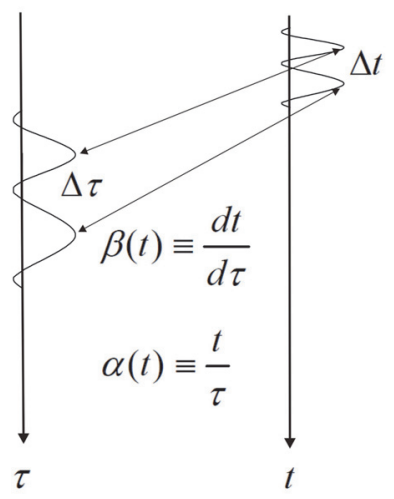

Frequency domain

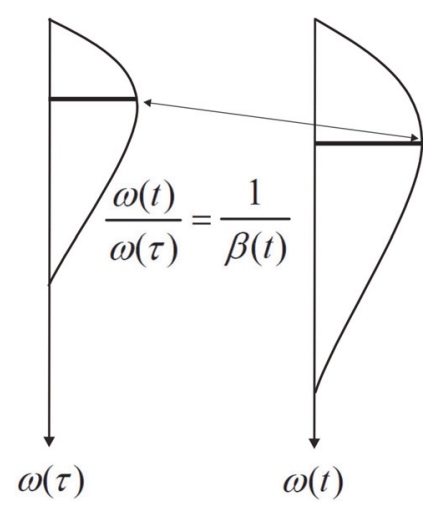

Figure 4. When the original PS- time $\tau$ is transformed to the PP-wave time $t$, the wavelet is squeezed, and the corresponding spectrum is stretched. The quantity $\alpha$ is the ratio of time $t$ and $\tau$, and $\beta$ is the compression rate. Spectrum stretch rate is $1 / \beta$. 
For a given $\gamma(t)$ model, we estimate the following two quantities. The first one is the ratio of the transformed time $t$ to the original time $\tau$, defined as

$$
\alpha(t) \equiv \frac{t}{\tau}=\frac{2}{1+\gamma(t)}
$$

Taking first-order differentiation produces another quantity,

$$
\beta(t) \equiv \frac{d t}{d \tau}=\frac{2}{1+\gamma(t)+t \gamma^{\prime}(t)},
$$

where $\gamma^{\prime}(t)$ is the differential of $\gamma(t)$ with respect to time $t$. This quantity measures the wavelet compression rate (to first order) in the PS-to-PP time transformation. The ratio of the instantaneous frequencies is inversely proportional to the wavelet compression rate as (Barnes, 1992)

$$
\frac{\omega(t)}{\omega(\tau)}=\frac{d \tau}{d t}=\frac{1}{\beta(t)}
$$

where $\omega(\tau)$ and $\omega(t)$ are the instantaneous frequency before and after transform respectively (Figure 4).

For amplitude correction, let us consider a local frequency spectrum $u_{P S}\left(\omega\left(t_{c}\right)\right)$ centered at time $t_{c}$. The corresponding PS-wave time is $\tau_{c}=t_{c} / \alpha\left(t_{c}\right)$. We modify this local frequency spectrum and make it equivalent to $u_{P S}\left(\omega\left(\tau_{c}\right)\right)$ in the following three operation steps: (1) finding $\gamma\left(t_{c}\right)$ and calculate $\beta\left(t_{c}\right)$ using equation 7, (2) resampling $\omega\left(t_{c}\right) \rightarrow \omega\left(\tau_{c}\right)$, based on equation 8 , and (3) modifying the local frequency spectrum by a factor of $\left|\beta\left(t_{c}\right)\right|$. In summary we modify the local frequency spectrum within the Gabor transform time window by

$$
u_{P S}\left(\omega\left(t_{c}\right)\right) \rightarrow\left|\beta\left(t_{c}\right)\right| u_{P S}\left(\beta\left(t_{c}\right) \omega\left(t_{c}\right)\right)=u_{P S}\left(\omega\left(\tau_{c}\right)\right)
$$

After correcting all of the local frequency spectra (both phase and amplitude) over the entire time range, we perform an inverse Gabor transform to re-produce a wavelet corrected PS-wave trace in time $t$.

For the synthetic example, Figure 5a compares the PP-wave trace, the original PS-wave trace before calibration, the PS-wave trace after compression, and the one with amplitude-spectrum correction. Figure $5 \mathrm{~b}$ compares the amplitude spectrum of the original PS-wave trace and the spectrum of the transformed PS-wave trace without frequency-spectrum correction. In Figure 5c, the distorted amplitude spectrum in the event registration is now recovered, and the bandwidth is compressed into the same range with the original PS-wave trace. The resultant wavelets (trace 4 of Figure 5a) are close to those in the original PS-wave seismic trace (trace 2 of Figure 5a). Figure 5d shows a zoomed in comparison of the second wavelet from each trace in Figure 5a.

Note the Nyquist frequency is $125 \mathrm{~Hz}$, the average $\beta$ value is about 0.67 in this example, and thus the corrected amplitude spectrum has valid frequencies only up to about $84 \mathrm{~Hz}$. The original high-frequency information (higher than $84 \mathrm{~Hz}$ ) would be lost during the waveform shrinking stage. This loss can be circumvented in practice if we upsample the data before the compression or have a smaller interval $\Delta t$ than $\Delta \tau$ during the trace compression. In the compression of Figure 5, we use an interval $\Delta t$ as a half of the original interval $\Delta \tau$. 


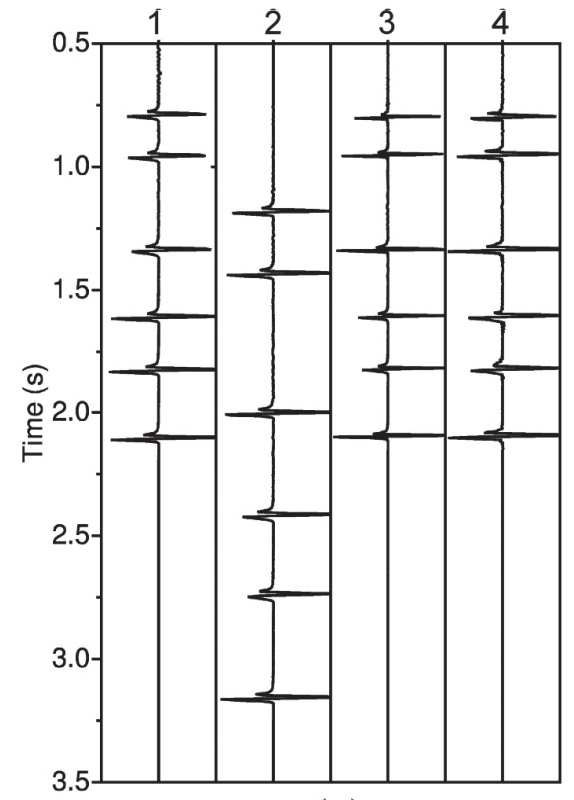

(a)

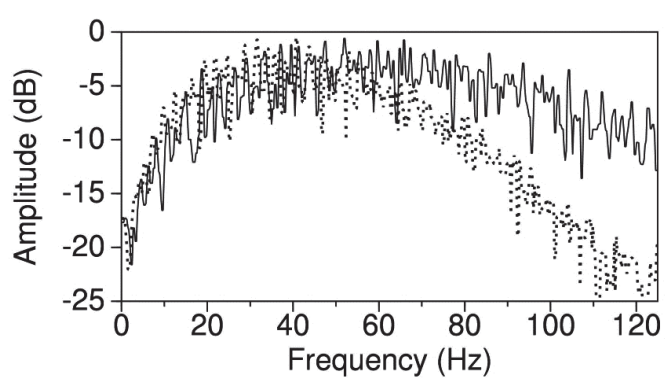

(b)

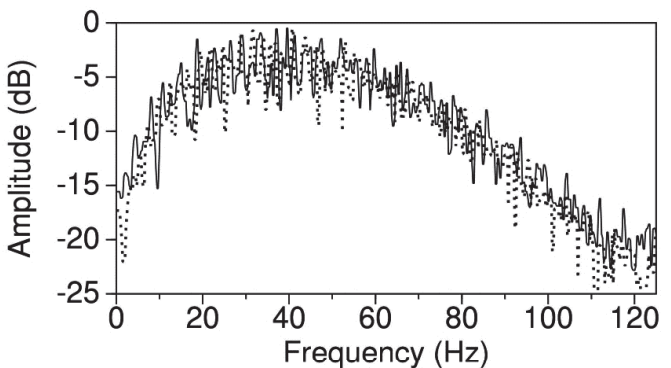

(c)

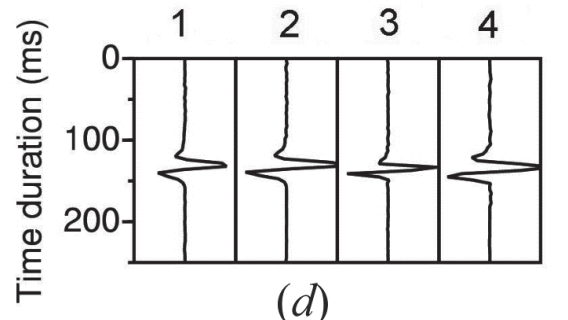

Figure 5. (a) The PP-wave trace (1), the original PS-wave trace (2), the PS-wave trace after event registration (3), and the PS-wave trace after amplitude-preserving event registration (4). (b) Amplitude spectra of a PS-wave trace before (dashed line) and after (solid line) event registration. (c) Amplitude spectra of the PS-wave trace before (dashed line) and after (solid line) event registration with amplitude correction. (d) Comparison of the zoomed-in wavelet (the second wavelet) from traces in Figure 5a.

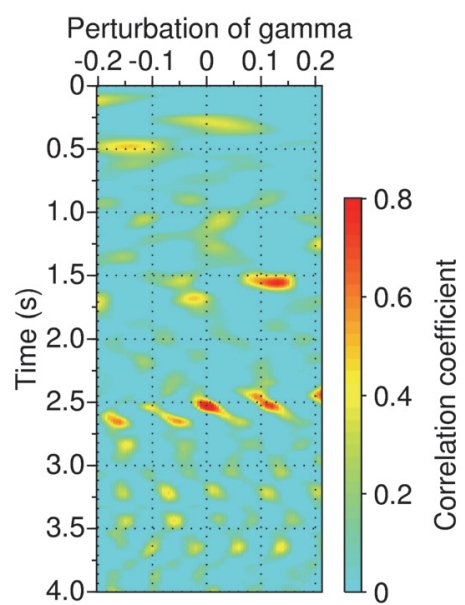

(a)

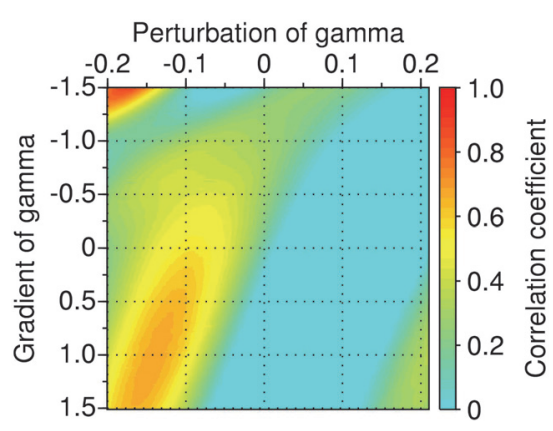

(b)

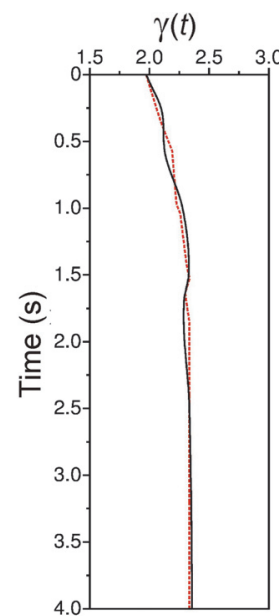

(c)

Figure 6. (a) PP- and PS-wave correlation-coefficient spectrum using time-variant $\gamma(t)$. (b) Two-dimensional analysis around $0.6 \mathrm{~s}$. The horizontal axis is the perturbation of local constant $\gamma_{c}$, and the vertical axis is the local gradient $d \gamma / d t$. (c) Updated $\gamma(t)$ function (solid black line) and the initial $\gamma(t)$ function (dashed red line). 


\section{Field data application}

We now apply the $\gamma(t)$ estimation and wavelet preservation methods to a real data example. For field seismic data, most likely, the PS-wave reflections cannot be easily distinguished from each other, and sometimes the signal-to-noise ratio is poor. Besides, the PS-wave events are often hard to recognise as distinct reflections; that might be true for PP waves as well. Therefore, we combine the previous perturbation method with a small interval analysis approach (Gaiser, 1996) to estimate the $\gamma(t)$ function in a layered fashion.

We first use the correlation-coefficient spectrum to analyze the hand-picked time-variant $\gamma(t)$ function with perturbations $\Delta \gamma$ (Figure 6a). When picking the $\gamma$ value at a time $t$, we pick a point with the smallest perturbation $\Delta \gamma$, if there are two or more ambiguous peaks. For example, we pick a relatively weak point at time $1.7 \mathrm{~s}$ with perturbation $\Delta \gamma=-0.04$, but not the strong one at time $1.51 \mathrm{~s}$ where shows perturbation $\Delta \gamma=0.1$. With an updated $\gamma(t)$ function, we can re-calculate the correlation spectrum with the improved image and re-test/validate the perturbations. However, there are always some intervals where we are not able to pick the $\gamma$ values confidently. For example, the correlation spectrum displays a zone from 0.5 to $0.7 \mathrm{~s}$ with relatively wide-spread correlation over the $\gamma$ perturbation.

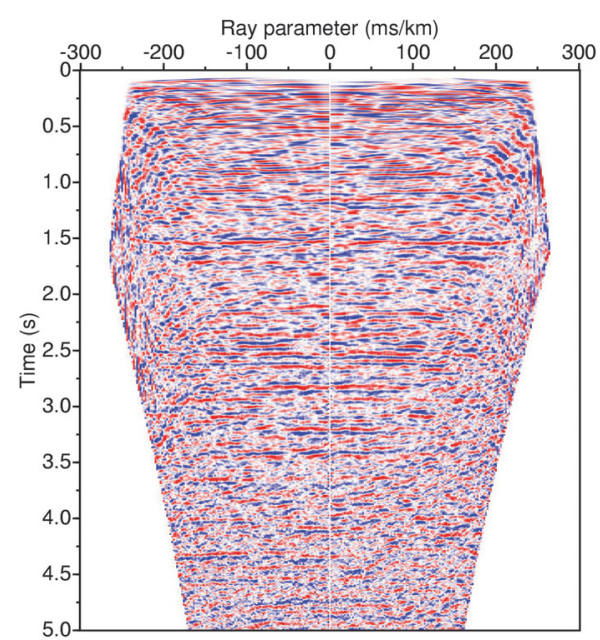

(a)

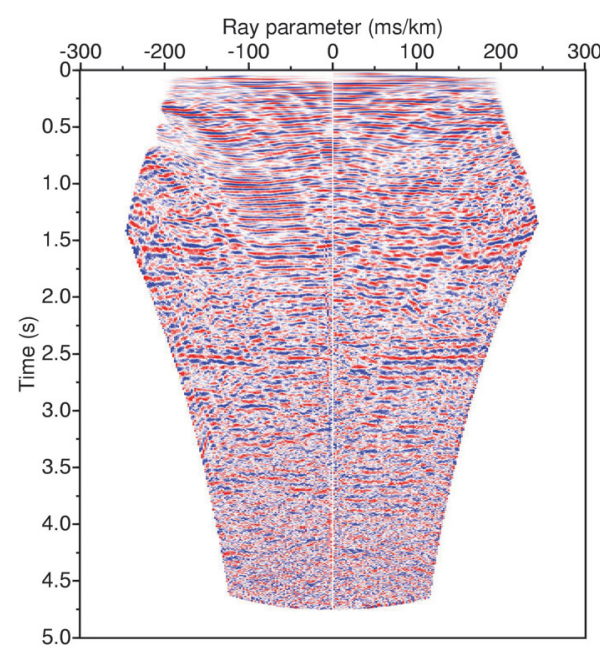

(c)

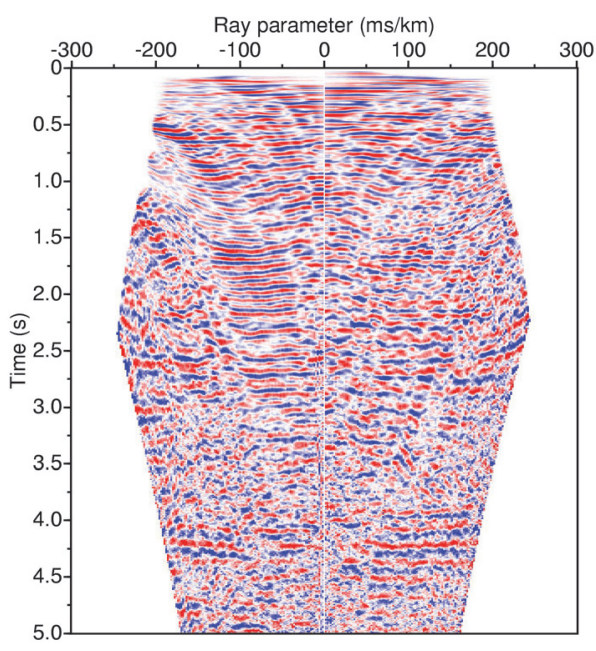

(b)

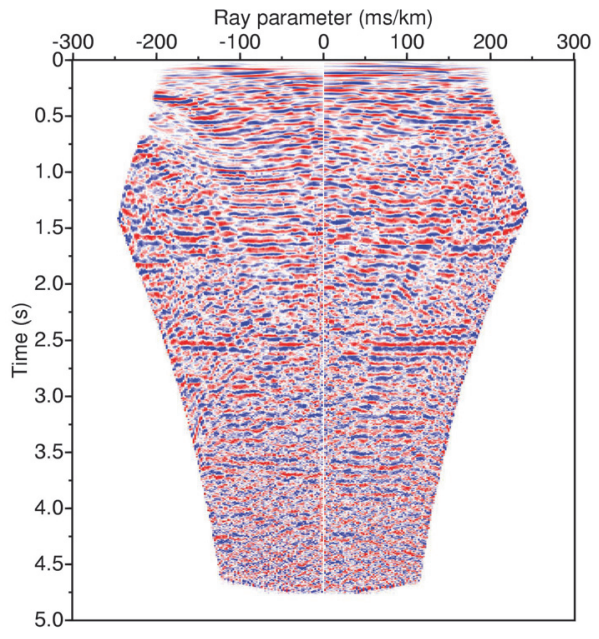

(d)

Figure 7. (a) PP-wave CIP gather. (b) PS-wave CIP gather. (c) Transformed PS-wave CIP gather without the frequency spectrum correction. (d) Transformed $P S$-wave CIP gather after the spectrum correction. 


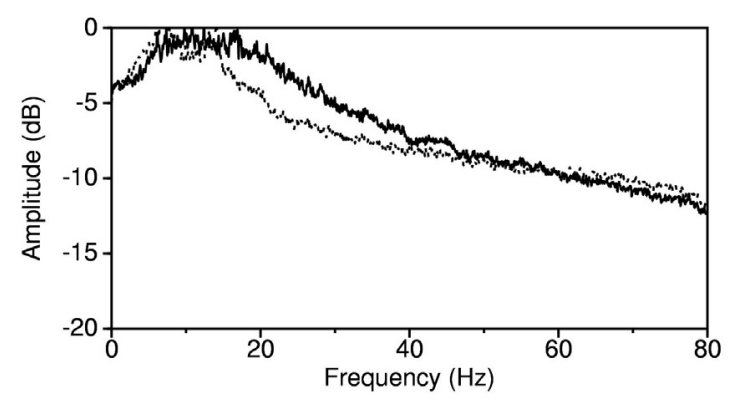

(a)

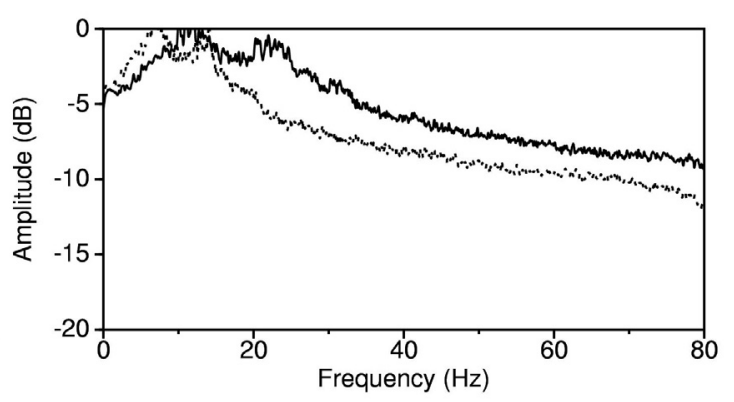

(b)

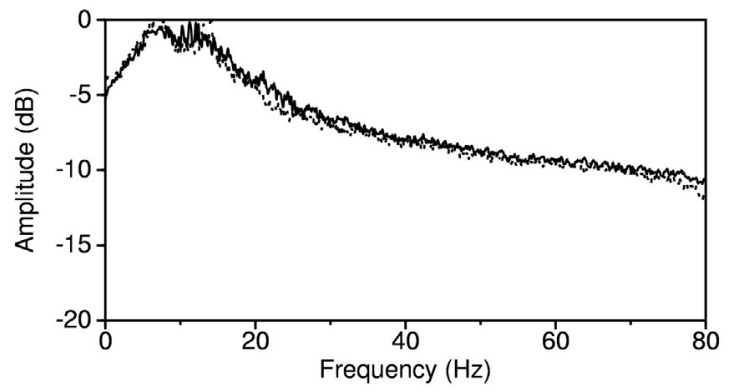

(c)

Figure 8. (a) Amplitude spectra of PP-wave (solid line) and PS-wave (dashed line) CIP gathers. (b) Amplitude spectra of a PS-wave gather before (dashed line) and after (solid line) the event registration (without spectrum correction). (c) Amplitude spectra of the PS-wave gather before (dashed line) and after (solid line) the event registration with spectrum correction.

To further improve the precision, we conduct the correlation analysis including not only the perturbation but also the gradient of $\gamma(t)$. We scan the $\gamma(t)$ using the following expression:

$$
\gamma(t)=\gamma_{\mathrm{c}}+\Delta \gamma+\frac{d \gamma}{d t}\left(t-t_{\mathrm{c}}\right)
$$

where $t_{\mathrm{c}}$ is the central time of the analysis window, $\gamma_{\mathrm{c}} \equiv \gamma\left(t_{\mathrm{c}}\right)$ is a constant within the window, $\Delta \gamma$ is the perturbation to the constant $\gamma_{c}$, and $d \gamma / d t$ is the local gradient. Figure $6 \mathrm{~b}$ is an example of a scanning window centred at $0.6 \mathrm{~s}$. The perturbation to the constant $\gamma_{\mathrm{c}}$ is from -0.2 to 0.2 , and the local gradient $d \gamma / d t$ from -1.5 to $1.51 / \mathrm{s}$. It reveals that in a small time interval centred at $0.6 \mathrm{~s}$, the value of $\gamma(t)$ is about $\gamma_{\mathrm{c}}-0.13$, with an increasing rate of 1.0 per second. Figure $6 \mathrm{c}$ compares the updated $\gamma(t)$ function (solid black line) with the initial $\gamma(t)$ function (dashed red line).

Figures $7 \mathrm{a}$ and $7 \mathrm{~b}$ are PP- and PS-wave CIP gathers generated from prestack time migration. Figures $7 \mathrm{c}$ and $7 \mathrm{~d}$ directly compare the transformed PS-wave CIP gathers without and with amplitude-spectrum correction. We can see that the strong reflections are well calibrated to the PP-wave events in Figure 7a.

Figure 8a compares the amplitude spectra of the PP-wave and PS-wave CIP gathers (Figures $7 \mathrm{a}$ and $7 \mathrm{~b}$ ), and evidently shows the difference in the dominant frequencies. Figure $8 \mathrm{~b}$ compares the original PS-wave amplitude spectrum with the spectrum of transformed PS-wave gather (without spectral correction). It reveals that the dominant frequency of the time transformed CIP gather shifts to a higher value, which is close to the dominant frequency of the PP-wave gather shown in Figure 8a. After the frequency spectrum correction, the calibrated and the original PS-wave CIP gathers have a similar dominant frequency in the amplitude spectra, as shown in Figure 8c. Each amplitude spectrum in Figure 8 is the average of the amplitude spectra of all individual traces in a CIP gather. 


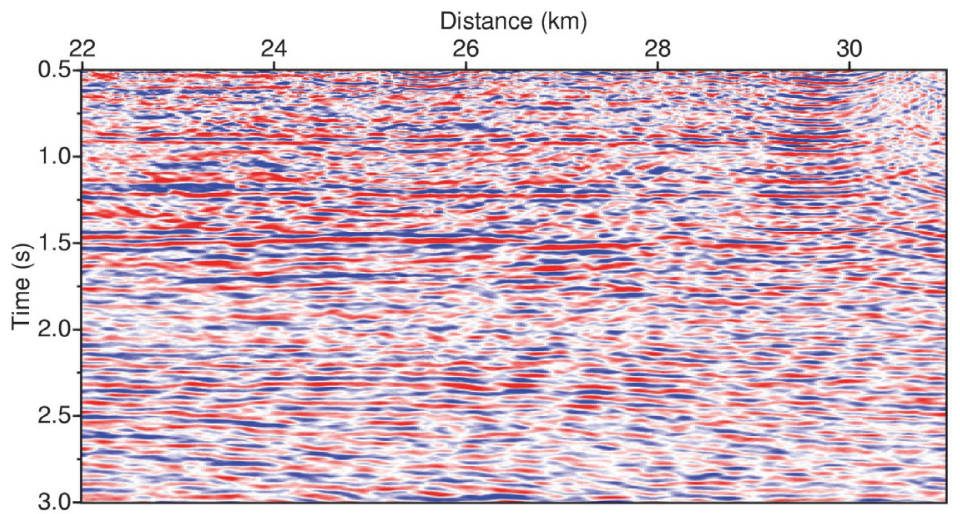

(a)

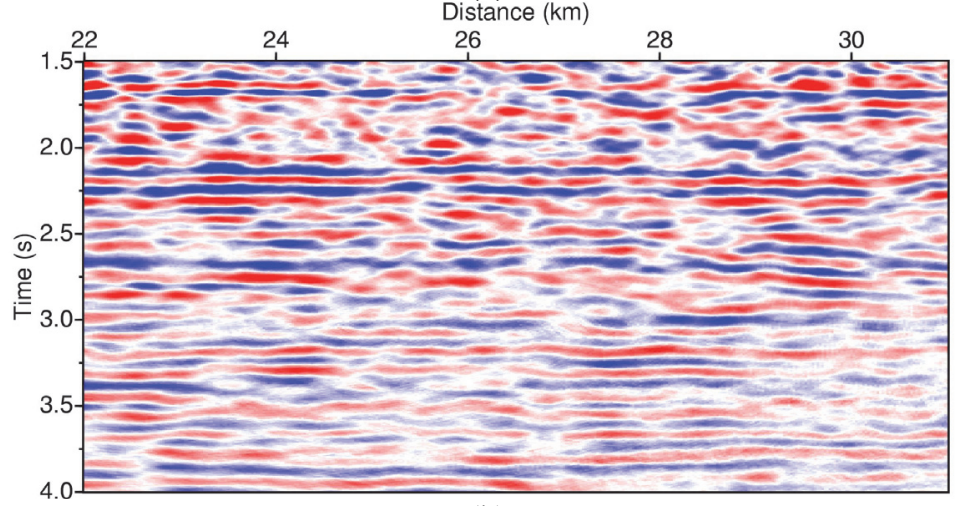

(b)

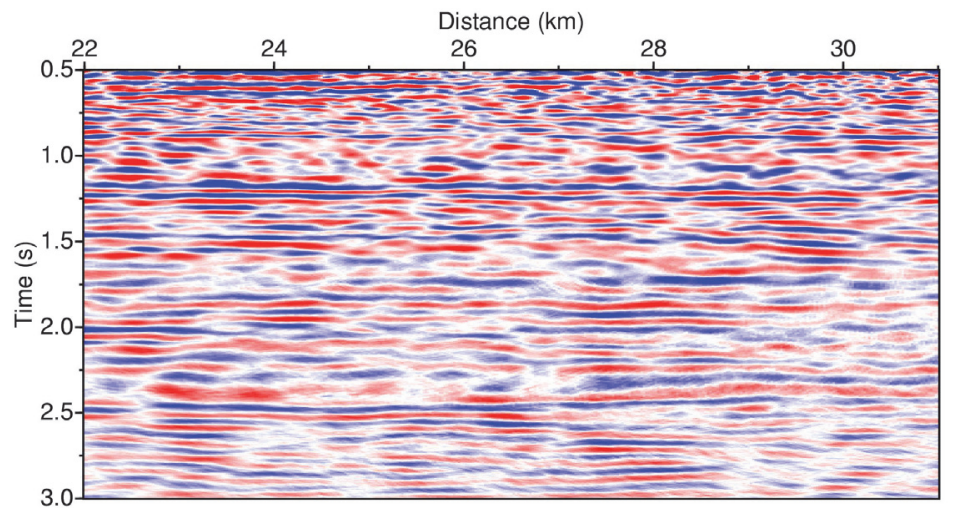

(c)

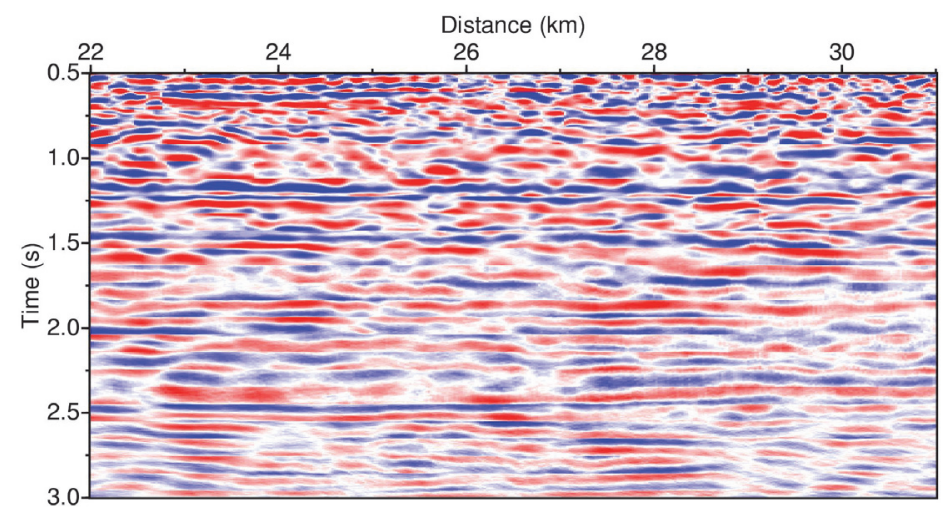

(d)

Figure 9. Amplitude preserved event calibration on the constant ray-parameter profile (CRP) with $p=$ $150 \mathrm{~ms} / \mathrm{km}$. (a) A PP-wave CRP. (b) The corresponding PS-wave CRP. (c) Transformed PS-wave CRP without the spectrum correction. (d) Transformed $P S$-wave CRP with the spectrum correction. 
Figure 9 shows the example of amplitude preserved event calibration results on the constant ray-parameter profile (CRP) with $p=150 \mathrm{~ms} / \mathrm{km}$. Figures $9 \mathrm{a}$ and $9 \mathrm{~b}$ gives a PP-wave CRP from 0.5 to $3 \mathrm{~s}$, and the corresponding PS-wave CRP from 1.5 to $4.0 \mathrm{~s}$. Figures $9 \mathrm{c}$ and $9 \mathrm{~d}$ are the PS-wave CRPs transformed to the PP time without and with the frequency preservation, respectively. We can observe that the resolution is compromised on the seismic profile due to the narrow frequency bandwidth after the frequency preservation processing. However, preserving PS-wave amplitudes during the calibration process is important in seismic ray-impedance inversion workflow for quantitative estimation of reservoir properties. We may of course move an extra step in an alternative direction to match the wavelengths of $\mathrm{PP}$ and PS reflections for stratigraphic interpretation.

\section{Conclusions}

This paper presents a procedure for the PS- to PP-wave calibration, which involves the estimation of the P- to S-wave velocity ratio $\gamma$, followed by a frequency spectrum correction to preserve the wavelets in a transformed PS-wave trace. While manual picking on PP- and PS-wave gathers is used as an initial $\gamma(t)$ model, we perform time-variant event-correlation analysis with perturbations to determine the $\gamma(t)$ function for the entire trace. We then refine it further by expanding the search to include the gradient of the local $\gamma(t)$ trend at chosen target locations.

The transformation to the PP-wave two-way time squeezes wavelets in a PS-wave trace. Wavelet compression means stretching in the frequency spectrum, and this spectral stretching is time variant, depending on $\gamma(t)$. Therefore, we implement time-frequency spectrum restoration in the Gabor-transform domain, to preserve the reflection wavelets to that in the original PS-wave trace. The PS-wave trace after PP-time compression with wavelet preservation may be used in seismic inversion jointly with PP-waves, as reflections at the same time in a pair of PP and PS traces with constant ray-parameter value originated from the same reflection point both laterally and vertically (in pseudo depth).

\section{Acknowledgements}

We are grateful to the sponsors of the Centre for Reservoir Geophysics, Imperial College London, for supporting this research. F. Zhang was also partially funded by British Geological Survey through the University Collaboration scheme for his PhD studies.

\section{References}

Barnes, A. E., 1992, Another look at NMO stretch. Geophysics, 57, 749-51.

Behle, A., and Dohr G., 1985, Converted waves in exploration seismics, in Dohr, G., Ed., Seismic shear-waves, Part B, Applications. Geophysical Press. vol 15B, 178-223.

Castagna, J. P., Batzle M. L., and Eastwood R. L., 1985, Relationships between compressional- and shear-wave velocities in elastic silicate rocks. Geophysics, 50, 571-81.

Fomel, S., 2007, Local seismic attributes. Geophysics 72, A29-33.

Gaiser, J. E., 1996, Multicomponent $\mathrm{V}_{\mathrm{p}} / \mathrm{V}_{\mathrm{S}}$ correlation analysis. Geophysics 61, 1137-49.

Garotta, R., 1985, Observation of shear waves and correlation with P events, in Dohr, G., Ed., Seismic shear-waves, Part B, Applications. Geophysical Press, Vol 15B, 1-86.

Li, X., Yuan J., Ziolkowski A., and Strijbos F., 1999, Estimating $\mathrm{V}_{\mathrm{P}} / \mathrm{V}_{\mathrm{S}}$ ratio from converted waves: a 4C case example. 61st Annual EAGE Meeting Extended Abstracts, P066.

Nickel, M., and Sonneland L., 2004, Automated PS to PP event registration and estimation of a high-resolution $\mathrm{V}_{\mathrm{p}}-\mathrm{V}_{\mathrm{S}}$ ratio volume. $74^{\text {th }}$ SEG Meeting Expanded Abstracts, 869.

Nickel, M., and Sonneland L., 2005, 3D high-resolution Vp/Vs inversion: Experiences from recent case studies. EAGE/SEG Research Workshop in Pau, 'Multicomponent Seismic - Past, Present and 
Future', Extended Abstracts.

Ogiesoba, O. C., and Stewart R. R., 2003, $\mathrm{V}_{\mathrm{P}} / \mathrm{V}_{\mathrm{S}}$ from multicomponent seismic data and automatic PS to PP time mapping. $73^{\text {rd }}$ SEG Meeting Expanded Abstracts, 789.

Thomsen, L., 1999, Converted-wave reflection seismology over inhomogeneous, anisotropic media. Geophysics, 64, 678-90.

Wang, Y., 2003, Seismic Amplitude Inversion in Reflection Tomography. (Amsterdam: Elsevier)

Wang, Y., 2006, Inverse $Q$-filter for seismic resolution enhancement. Geophysics 71, V51-60.

Wang, Y., 2008, Seismic Inverse Q filtering. (Oxford: Blackwell Publishing)

Zhang, J., and Li X., 2004, P-wave and converted wave event correlation by converted wave moveout analysis. $66^{\text {th }}$ EAGE Meeting Extended Abstracts, Z-99.

Zhang F., Wang Y., and Li X., 2009, Mixed-phase wavelet estimation using unwrapped phase of bispectrum. $71^{\text {st }}$ EAGE Meeting Extended Abstracts, P-81. 